\title{
THE REAL REASON FOR RIVESTS PHENOMENON
}

\author{
Don Coppersmith \\ IBM Research \\ Yorktown Heights, NY 10598
}

Burt Kaliski, Ronald Rivest and Alan Sherman [Crypto 85] noticed a short cycle in their experiments with weak keys in DES. We explain this in terms of fixed points (messages which are left unchanged by encipherment). We predict similar short cycles using semi-weak keys. We indicate bow Rivest et als experimental setup can be used to show that the group of permutations of message space, generated by DES encryptions, is a large group.

Notation: Let $E_{K} X$ denote the cipbertext resulting from DES-encrypting the cleartext $X$ under the key $K$. Similarly let $D_{K^{X}}$ represent decryption. Let 0 denote the key of all 0 's, and 1 the key of all 1 's. Let the input to DES (the cleartext) be broken into balves $M_{0}, M_{1}$. On round $i, 1 \leq i \leq 16$, we compute some function $f$ of the 48 key bits $K_{i}$ and the 32 message bits $M_{i+}$ add this 32 -bit quantity to $M_{i-1}$ bitwise, and obtain $M_{i+1}$. So $M_{i+1}=M_{i-1}+f\left(K_{i}, M_{i}\right)$, and $M_{i-1}=M_{i+1}+f\left(K_{i}, M_{i}\right)$. The cipbertext is the pair $M_{17}, M_{16}$. (Notice the order of indices, which is correct.)

The keys 0 and 1 (and two others) are known to be weak keys [Davies, Crypto 82] in the sense that the 48 key bits $K_{i}$ entering into the computation on round $i$ are the same for each round $i: K_{i}=K_{j}$ One consequence of this is that $E_{0}$ is an involution: $E_{0} X=D_{0} Y$, so that $E_{0}^{2} X=X$.

A sew consequence of being a weak key is that $E_{0}$ has $2^{32}$ fixed points, i.e. messages $Y$ for which $E_{0} Y=Y$. Indeed, for some message $Y$, suppose that $K_{8}=M_{9}$. (There are $2^{32}$ such values of $Y$.) Then

$$
M_{7}=M_{9}+f\left(K_{8}, M_{8}\right)=M_{8}+f\left(K_{9}, M_{9}\right)=M_{10}
$$

Continuing, we find $M_{6}=M_{11}, \ldots, M_{1}=M_{16}, M_{0}=M_{17}$, and $Y=\left(M_{0}, M_{1}\right)=\left(M_{17}, M_{16}\right)-E_{0} Y$. In fact this is the only way a fixed point can arise for any weak key.

Now pick a random starting message $X$, and alternately apply $E_{0}$ and $E_{1}$. Continue until you retum to the starting point $X:\left(E_{1} E_{0}\right)^{N} X=X$. In Rivest et $\sigma \Gamma_{\text {s }}$ experiment, $N$ turned out to be around $2^{32}$. Indeed, suppose that for some $I<N,\left(E_{1} E_{0}\right)^{I} X=Y$ and $Y$ is a fixed point of $E_{0}$. Then the next application of $E_{0}$ leaves $Y$ unchanged, so that $E_{0}\left(E_{1} E_{0}\right)^{I} X=Y$. On the next application of $E_{1}$, we find

$$
\left(E_{1} E_{0}\right)^{I+1} X=E_{1} E_{0}\left(E_{1} E_{0}\right)^{I} X=E_{1} Y=D_{1} Y=D_{1} E_{1} E_{0}\left(E_{1} E_{0}\right)^{I-1} X=E_{0}\left(E_{1} E_{0}\right)^{I-1} X
$$

Continuing, for $J \leq I, \quad\left(E_{1} E_{0}\right)^{I+J} X=E_{0}\left(E_{1} E_{0}\right)^{I-J} X$, and we are just retracing our steps. This is because both $E_{0}$ and $E_{1}$ are involutions. We continue until, for some $J>I, \quad\left(E_{1} E_{0}\right)^{I+J} X=Z$ and $Z$ is another fixed point of $E_{0}$. (We could also find fixed points of $E_{1}$.) We again ead up retracing our steps, until we retum to the starting value $X$. 
The cycle length $N$ is approximately the number of trials we needed to find two fixed points (of either $E_{0}$ or $E_{1}$.) Since these fixed points are plenciful $\left(2^{32}\right.$ out of $2^{64}$, or 1 out of $\left.2^{32}\right)$, the expected value of $N$ is $2^{32}$, in close agreement with Rivest et af's results.

In a similar vein, suppose $K$ is alternating $010101 \ldots$ or $101010 \ldots$ in eacb key balf (a special case of the "semiweak" keys [Davies]). Let $\bar{Z}$ denote the complement of $Z$. Then we find that $E_{K}=D_{\bar{K}}$; there are $2^{32}$ values $Y$ for which $E_{K} Y=\bar{Y}$ (namely those for which $M_{g}=\bar{M}_{9}$ ); and for a random $\mathrm{X}$ we expect to find that $E_{K}^{N} X=X$ for $N \simeq 2^{33}$.

Finally, for different starting values $X_{i}$, we expect to find different cycle lengths $N_{i}$. Consider the subgroup $G$ of the group of permutations on message space $S_{2} 64$ generated by the DES encryptions $E_{K}, K \in Z_{2}^{56}$. Each $N_{j}$ divides the size of $G$. Run either of the above experiments several times, finding different values $N_{i}$ corresponding to $E_{1} E_{0}$ or to $E_{K}$ for one of the four alternating semiweak keys $K$. Eacb experiment takes a few days. Then the least common multiple $\mathrm{lm}\left(N_{1}, N_{2}, \ldots, N_{i}\right)$ divides the order of the group, and thus provides a lower bound. So the experiments, which were designed to detect a small group size $\left(|G|<2^{70}\right.$ ?) might be used to show a large group size $\left(|G|>2^{300} ?\right)$. 